\title{
Unexpected Migration and Oxidative Cyclization of Substituted 2-Acetophenone Triflates under Basic Conditions: Synthetic and Mechanistic Insights
}

Jotham W. Coe,* Krista E. Bianco, Brian P. Boscoe, Paige R. Brooks, Eric D. Cox, and Michael G. Vetelino

Pfizer Global Research and Development, Groton Laboratories, Pfizer Inc., Groton, CT 06340

\section{Table of Contents}

General Experimental

Synthesis of 2-Acetophenone Triflates

S3

General method for the preparation of Weinreb amides from carboxylic

acids

Preparation of Cyclopent-3-enecarboxylic acid methoxy-methyl-amide

General method for the preparation of substituted 2-Methoxyacetophenones

Preparation of cyclopent-3-enyl-(2-methoxy-phenyl)-methanone (2-

methoxyacetophenone (1g anisole)

2-Cyclopropyl-1-(2-methoxy-phenyl)-ethanone (4) (1c anisole) $\quad S 4$

1-(2-Methoxy-phenyl)-hept-6-en-1-one (1d anisole)

1-(2-Methoxy-phenyl)-3,7-dimethyl-oct-6-en-1-one (1e anisole)

Cyclopentyl-(2-methoxy-phenyl)-methanone (1f anisole)

General method for the preparation of substituted 2-hydroxyacetophenones

Preparation of cyclopent-3-enyl-(2-hydroxy-phenyl)-methanone (1g phenol)

2-Cyclopropyl-1-(2-hydroxy-phenyl)-ethanone (1c phenol)

1-(2-Hydroxy-phenyl)-hept-6-en-1-one (1d phenol)

1-(2-Hydroxy-phenyl)-3,7-dimethyl-oct-6-en-1-one (1e phenol)

Cyclopentyl-(2-hydroxy-phenyl)-methanone (1f phenol)

General method for the preparation of substituted 2-trifluoromethane

Preparation of trifluoro-methanesulfonic acid 2-(cyclopent-3-enecarbonyl)-

Trifluoro-methanesulfonic acid 2-acetyl-phenyl ester (1a)

Trifluoro-methanesulfonic acid 2-propionyl-phenyl ester (1b) $\quad S 7$

Trifluoro-methanesulfonic acid 2-(2-cyclopropyl-acetyl)-phenyl ester (1c) $\quad S 7$

Trifluoro-methanesulfonic acid 2-hept-6-enoyl-phenyl ester (1d)

Trifluoro-methanesulfonic acid 2-(3,7-dimethyl-oct-6-enoyl)-phenyl ester (1e)

Trifluoro-methanesulfonic acid 2-cyclopentanecarbonyl-phenyl ester (1f) $\quad S 8$ Trifluoro-methanesulfonic acid 2-methyl-2-propionyl-phenyl ester (1h) Migration reaction $(1 \rightarrow 3)$ 
Preparation of trifluoro-methanesulfonic acid 1-(2-hydroxy-phenyl)-propenyl $S 8$ - $S 9$ ester (3b) $\underline{t-B u O K / T H F}$ conditions

Preparation of trifluoro-methanesulfonic acid 1-(2-hydroxy-phenyl)-vinyl $S 9$ ester (3a) DBU/DMF conditions

Preparation of trifluoro-methanesulfonic acid 1-(2-hydroxy-phenyl)-vinyl $S 9$ ester (3a) KOAc/DMF conditions

Preparation of trifluoro-methanesulfonic acid 1-(2-hydroxy-phenyl)-vinyl $S 10$ ester (3a) LHMDS/THF conditions

Trifluoro-methanesulfonic acid 2-cyclopropyl-1-(2-hydroxy-phenyl)-vinyl S10 ester (3c)

Cyclization reaction $(1 \rightarrow 2) \quad S 10$

Preparation of 2,2-dimethyl-benzofuran-3-one (2h) $\quad S 10$

Preparation of 2-methyl-benzofuran-3-one (2b) S11

Preparation of 2-methyl-benzofuran-3-one (2b) S11

Preparation of 2-methyl-benzofuran-3-one (2b) S11

Preparation of 2-methyl-benzofuran-3-one (2b) and conversion of trifluoro- $\quad$ S11-S12 methanesulfonic acid salt to trifluoromethanesulfonylmethyl-benzene

Preparation of 2-pent-4-enyl-benzofuran-3-one (2d) S12

Preparation of 2-(1,5-dimethyl-hex-4-enyl)-benzofuran-3-one (2e) $\quad S 12$

Preparation of 2,2-spiropentanylbenzofuran-3-one (2f) S12

Preparation of 2,2-spirocyclopentene benzofuran-3-one (2g) $\quad S 13$

Reference 13: Spiro product from Conia Cyclization $\quad$ S13-S14

Preparation of Trifluoro-methanesulfonic acid 2-cyclopropyl-1-(2-methoxy- $\quad S 14$ phenyl)-vinyl ester (5)

$\begin{array}{ll}\text { Stability study of } 4 \text { and } 5 . & \text { S14-S15 }\end{array}$

General: Unless otherwise noted, all materials were purchased from commercial sources. Anhydrous solvents DMF, THF, $\mathrm{CH}_{2} \mathrm{Cl}_{2}$ and $\mathrm{CH}_{3} \mathrm{CN}$ were handled under dry nitrogen atmosphere. TLC was performed with EM separations technology silica gel $F_{254}$. Silica gel chromatography was carried out with J. T. Baker 40- $\mu \mathrm{m}$ silica gel according to Still's procedure (Still, W. C., Kahn, M., Mitra, A. J. Org. Chem. 1978, 43, 2923.) All glassware was flame dried under dry nitrogen purge before use. ${ }^{1} \mathrm{H}$ NMR spectra were collected at $400 \mathrm{MHz}$ with residual $\mathrm{CHCl}_{3}$ as standard (7.26 ppm) and ${ }^{13} \mathrm{C}$ NMR collected at $100 \mathrm{MHz}$. Not all ${ }^{13} \mathrm{C}$ NMR resonances were recorded beyond $200 \mathrm{ppm}$ and most $\mathrm{CF}_{3}$ resonances were not well resolved. GC-MS and LC-MS are reported with observed parent ions and the percentage of all chromatographically observed peaks measured by 
TIC (total ion count). Degassing and deoxygenation are used interchangeably herein, and refer to evacuation $(\sim 20 \mathrm{~mm}) /$ nitrogen purge three or more times of substrate and solvent solutions prior to addition of base. All spectroscopic data for known compounds was in complete accord with literature values. For yields see Tables 1 and 2 and below.

\section{Synthesis of 2-Acetophenone Triflates}

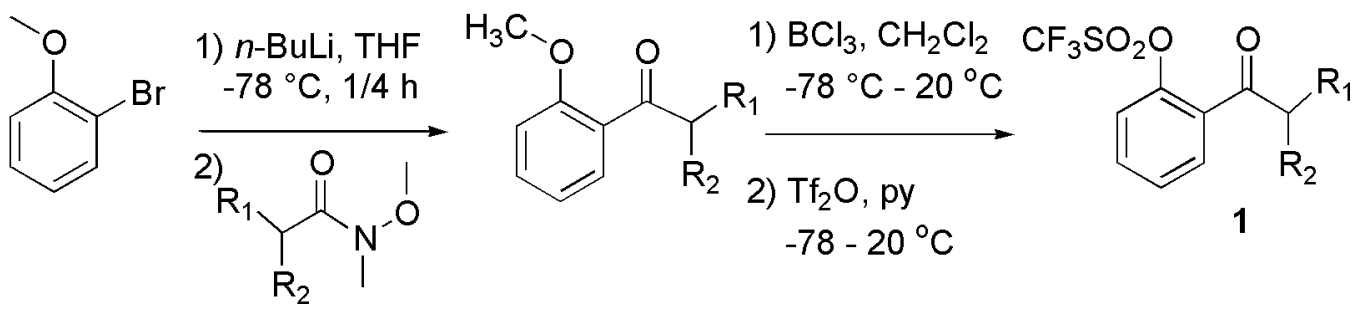

\section{General method for the preparation of Weinreb amides from carboxylic acids} Preparation of Cyclopent-3-enecarboxylic acid methoxy-methyl-amide

Cyclopent-3-enecarboxylic acid $(5.0 \mathrm{~g}, 44.6 \mathrm{mmol})$ in $\mathrm{CH}_{2} \mathrm{Cl}_{2}(150 \mathrm{~mL})$ was treated with carbonyl diimidazole $(7.96 \mathrm{~g}, 49.1 \mathrm{mmol})$ in portions. After $\sim 3 / 4 \mathrm{~h}$, the resulting solution was treated with $\mathrm{N}, \mathrm{O}$-dimethylhydroxylamine hydrochloride $(4.8 \mathrm{~g}, 49.1 \mathrm{mmol})$ and the mixture was stirred for 4-18 h. The reaction was quenched with $1 \mathrm{~N}$ aqueous $\mathrm{HCl}$ solution $(60 \mathrm{~mL})$, shaken and the layers were separated. The aqueous layer was extracted with $\mathrm{CH}_{2} \mathrm{Cl}_{2}(2 \times 20 \mathrm{~mL})$. The combined organic layer was washed with $1 \mathrm{~N}$ aqueous $\mathrm{HCl}$ solution $(20 \mathrm{~mL}), \mathrm{H}_{2} \mathrm{O}(2 \times 25 \mathrm{~mL}), 50 \%$ saturated aqueous $\mathrm{Na}_{2} \mathrm{CO}_{3}$ solution/saturated aqueous $\mathrm{NaCl}$ solution $(25 \mathrm{~mL})$ and dried through a cotton plug. The filtrate was diluted with EtOAc to $\sim 10 \% \mathrm{EtOAc} / \mathrm{CH}_{2} \mathrm{Cl}_{2}$ and filtered through a silica pad $(3 \mathrm{x} 5 \mathrm{~cm}$ ) eluting with $10 \%$ EtOAc/ $\mathrm{CH}_{2} \mathrm{Cl}_{2}$ to remove baseline color. Concentration affords a liquid $(6.63 \mathrm{~g}$, 96\%). (TLC 10\%EtOAc/ $\mathrm{CH}_{2} \mathrm{Cl}_{2} \mathrm{R}_{\mathrm{f}} 0.56$ ); ${ }^{1} \mathrm{H}$ NMR $\left(\mathrm{CDCl}_{3}\right) \delta 5.64$ (br s, 2H), 3.69 (s, 3H), 3.47 (m, 1H), 3.19 (s, 3H), 2.61 (m, 4H); GS-MS m/z $155\left(\mathrm{M}^{+}, 100 \%\right)$.

This procedure was used to prepare hept-6-enoic acid methoxy-methyl-amide, 3,7-dimethyl-oct-6-enoic acid methoxy-methyl-amide (citronellic acid methoxy-methylamide) and 2-cyclopropyl-N-methoxy-N-methyl-acetamide from the corresponding commercial carboxylic acids. 


\section{General method for the preparation of substituted 2-Methoxyacetophenones}

Preparation of cyclopent-3-enyl-(2-methoxy-phenyl)-methanone

methoxyacetophenone (1g anisole) (For a discussion of halogen-metal exchange, see: Parham, W. E.; Bradsher, C. K. Acc. Chem. Res. 1982, 15, 300-305.)

2-Bromomethoxybenzene $(4.0 \mathrm{~g}, 21.4 \mathrm{mmol})$ was stirred in THF $(30 \mathrm{~mL})$ under $\mathrm{N}_{2}$ at -78 ${ }^{\circ} \mathrm{C}$. $n$-BuLi $(9.4 \mathrm{~mL}, 2.5 \mathrm{M}$ in hexanes, $23.5 \mathrm{mmol})$ was added to the resulting solution over $10 \mathrm{~min}$. After stirring $10 \mathrm{~min}$., the yellow solution was treated with cyclopent-3enecarboxylic acid methoxy-methyl-amide (3.75 g, $21.4 \mathrm{mmol})$ in THF (50 mL) over 10 min. After $1 \mathrm{~h}$ at $-78{ }^{\circ} \mathrm{C}$, the mixture was allowed to warm to ambient temperature. After stirring an additional $1 \mathrm{~h}$, the mixture was poured into $10 \%$ aqueous $\mathrm{HCl}$ solution $(40 \mathrm{~mL})$ and stirred for $30 \mathrm{~min}$. The layers were separated and the aqueous layer extracted with $\mathrm{Et}_{2} \mathrm{O}(3 \times 30 \mathrm{~mL})$. The organic layer was washed with $\mathrm{H}_{2} \mathrm{O}(50 \mathrm{~mL})$, saturated aqueous $\mathrm{NaHCO}_{3}$ solution $(30 \mathrm{~mL})$, dried $\left(\mathrm{Na}_{2} \mathrm{SO}_{4}\right)$, filtered and concentrated to an oil. Purification by chromatography on silica gel eluting with $10 \%$ EtOAc/hexanes provided an oil (4.1 g, 95\%). ${ }^{1} \mathrm{H}$ NMR (400 MHz, $\left.\mathrm{CDCl}_{3}\right) \delta .7 .57$ (dd, J = 7.9, $\left.1.7 \mathrm{~Hz}, 1 \mathrm{H}\right), 7.40$ (ddd, J = 8.3, 7.5, 2.0 Hz, 1H), 6.96 (dt, J = 7.5, $0.9 \mathrm{~Hz}, 1 \mathrm{H}), 6.92$ (d, J = 8.3 Hz, 1H), 5.62 (br s, 2H), 4.07 (m, 1H), 3.85 (s, 3H), $2.69-2.55$ (m, 4H); ${ }^{13} \mathrm{C}$ NMR (100 MHz, $\left.\mathrm{CDCl}_{3}\right) \delta 205.6,158.2,133.1,130.6,129.2,129.1,120.8,111.6,55.8,49.0,36.0$; GC-MS $m / z 202\left(\mathrm{M}^{+}, 100 \%\right)$; HRMS calcd. 203.1072 for $\mathrm{C}_{13} \mathrm{H}_{15} \mathrm{O}_{2}$ : obs. $m / z 203.1072(\mathrm{M}+1)^{+}$.

This procedure was used to prepare the following substituted 2-methoxy acetophenones: 2-Cyclopropyl-1-(2-methoxy-phenyl)-ethanone (4) (1c anisole)

${ }^{1} \mathrm{H}$ NMR $\left(400 \mathrm{MHz}, \mathrm{CDCl}_{3}\right) \delta 7.66(\mathrm{dd}, \mathrm{J}=7.7,1.8 \mathrm{~Hz}, 1 \mathrm{H}), 7.42(\mathrm{ddd}, \mathrm{J}=8.4,7.3,1.7$ Hz, 1H), 6.98 (ddd, J = 7.7, 7.3, 1.0 Hz, 1H), 6.93 (d, J = 8.4 Hz, 1H), 3.87 (s, 3H), 2.86 $(\mathrm{d}, \mathrm{J}=6.9 \mathrm{~Hz}, 2 \mathrm{H}), 1.08(\mathrm{~m}, 1 \mathrm{H}), 0.51(\mathrm{~m}, 2 \mathrm{H}), 0.10(\mathrm{~m}, 2 \mathrm{H})$.

1-(2-Methoxy-phenyl)-hept-6-en-1-one (1d anisole) was carried on directly in crude form.

\section{1-(2-Methoxy-phenyl)-3,7-dimethyl-oct-6-en-1-one (1e anisole)}

${ }^{1} \mathrm{H}$ NMR $\left(400 \mathrm{MHz}, \mathrm{CDCl}_{3}\right) \delta 7.59(\mathrm{dd}, \mathrm{J}=7.7,1.8 \mathrm{~Hz}, 1 \mathrm{H}), 7.42($ br t, J = 8.3 Hz, 1H), $6.98(\mathrm{br} t, \mathrm{~J}=7.2 \mathrm{~Hz}, 1 \mathrm{H}), 6.93(\mathrm{~d}, \mathrm{~J}=8.3 \mathrm{~Hz}, 1 \mathrm{H}), 5.07$ (t, J = 7.0 Hz, 1H), 3.87 (s, 3H), $2.98(\mathrm{dd}, \mathrm{J}=15.9,5.6 \mathrm{~Hz}, 1 \mathrm{H}), 2.73(\mathrm{dd}, \mathrm{J}=15.9,8.5 \mathrm{~Hz}, 1 \mathrm{H}), 2.07$ (m, 1H), 1.97 (m, 
2H), $1.65(\mathrm{~s}, 3 \mathrm{H}), 1.57(\mathrm{~s}, 3 \mathrm{H}), 1.34(\mathrm{~m}, 1 \mathrm{H}), 1.23(\mathrm{~m}, 1 \mathrm{H}), 0.90(\mathrm{~d}, \mathrm{~J}=6.6 \mathrm{~Hz}, 3 \mathrm{H}) ;{ }^{13} \mathrm{C}$ NMR $\left(100 \mathrm{MHz}, \mathrm{CDCl}_{3}\right) \delta 158.8,133.0,131.3,130.1,129.2,124.6,120.6,111.4,55.4$, 51.2, 37.2, 29.4, 25.7, 25.6, 19.9, 17.7; IR ( $\left.\mathrm{cm}^{-1}\right)$ 2961.1, 2921.7, 2851.3, 1674.5, 1597.1, 1484.8, 1462.7, 1436.6, 1294.4, 1244.6, 1162.4, 1025.4, 765.2; GC-MS m/z $260\left(\mathrm{M}^{+}\right.$, $100 \%)$.

Cyclopentyl-(2-methoxy-phenyl)-methanone (1f anisole)

${ }^{1} \mathrm{H}$ NMR $\left(400 \mathrm{MHz}, \mathrm{CDCl}_{3}\right) \delta 7.53(\mathrm{dd}, \mathrm{J}=7.6,1.8 \mathrm{~Hz}, 1 \mathrm{H}), 7.40$ (br t, J = 7.5 Hz, 1H), 6.99 - 6.92 (m, 2H), 3.87 (s, 3H), $3.68(\mathrm{~m}, 1 \mathrm{H}), 1.83(\mathrm{~m}, 4 \mathrm{H}), 1.67$ (m, 2H), 1.58 (m, 2H);

${ }^{13} \mathrm{C} \mathrm{NMR}\left(100 \mathrm{MHz}, \mathrm{CDCl}_{3}\right) \delta 205.8,158.0,132.6,129.9,129.8,120.6,111.4,55.6,51.2$, 29.6, 26.1; IR $\left(\mathrm{cm}^{-1}\right)$ 2953.4, 2867.3, 1670.9, 1596.7, 1484.3, 1464.0, 1436.3, 1358.9, 1284.2, 1245.1, 1206.4, 1023.6, 879.6, 755.6; GC-MS m/z $204\left(\mathrm{M}^{+}, 100 \%\right)$.

\section{General method for the preparation of substituted 2-hydroxyacetophenones}

Preparation of cyclopent-3-enyl-(2-hydroxy-phenyl)-methanone (1g phenol) (See Nagaoka, H.; Schmid, G.; Iio, H.; Kishi, Y. Tetrahedron Lett. 1981, 22, 899.)

Cyclopent-3-enyl-(2,5-dimethoxy-phenyl)-methanone (808 $\mathrm{mg}, 4.0 \mathrm{mmol}$ ) was stirred in $\mathrm{CH}_{2} \mathrm{Cl}_{2}(15 \mathrm{~mL})$ at $-78{ }^{\circ} \mathrm{C}$ under $\mathrm{N}_{2}$ and treated with boron trichloride $\left(\mathrm{BCl}_{3}\right)(5.0 \mathrm{~mL}$, $1 \mathrm{M} \mathrm{CH}_{2} \mathrm{Cl}_{2}$ solution, $5.0 \mathrm{mmol}$ ) over $5 \mathrm{~min}$. The mixture was allowed to warm to ambient temperature, stirred 20 min., poured slowly into $\mathrm{H}_{2} \mathrm{O}(30 \mathrm{~mL})$ and stirred for 30 min. Saturated aqueous $\mathrm{NaCl}$ solution $(15 \mathrm{ml})$ was added and the layers were separated and the aqueous layer was extracted with $\mathrm{CH}_{2} \mathrm{Cl}_{2}(2 \times 30 \mathrm{~mL})$. The combined organic layer was washed with $\mathrm{H}_{2} \mathrm{O}(3 \times 25 \mathrm{~mL})$, saturated aqueous $\mathrm{NaHCO}_{3}$ solution $(40 \mathrm{~mL})$, dried through a cotton plug and filtered through a silica pad to remove baseline color. Concentration affords an amber oil (740 mg, 98\%) which was used without additional purification. ${ }^{1} \mathrm{H}$ NMR $\left(400 \mathrm{MHz}, \mathrm{CDCl}_{3}\right) \delta 7.74(\mathrm{dd}, \mathrm{J}=7.9,1.7 \mathrm{~Hz}, 1 \mathrm{H}), 7.44(\mathrm{ddd}, \mathrm{J}=$ 8.7, 7.0, 1.7 Hz, 1H), 6.97 (dd, J = 8.3, 1.2 Hz, 1H), 6.95 (ddd, J = 7.9, 7.0, 1.2 Hz, 1H), 5.67 (br s, 2H), $4.09(\mathrm{~m}, 1 \mathrm{H}), 2.81-2.66(\mathrm{~m}, 4 \mathrm{H}) ;{ }^{13} \mathrm{C} \mathrm{NMR}\left(100 \mathrm{MHz}, \mathrm{CDCl}_{3}\right) \delta 208.1$, 163.3, 136.3, 130.4, 129.1, 119.3, 119.1,118.9, 43.9, 36.7; GC-MS m/z $188\left(\mathrm{M}^{+}, 100 \%\right)$.

\section{This procedure was used to prepare the following substituted 2-hydroxy acetophenones:}




\section{2-Cyclopropyl-1-(2-hydroxy-phenyl)-ethanone (1c phenol)}

${ }^{1} \mathrm{H}$ NMR $\left(400 \mathrm{MHz}, \mathrm{CDCl}_{3}\right) \delta 7.70(\mathrm{dd}, \mathrm{J}=8.1,1.7 \mathrm{~Hz}, 1 \mathrm{H}), 7.45(\mathrm{ddd}, \mathrm{J}=8.5,7.2,1.6$ $\mathrm{Hz}, 1 \mathrm{H}), 6.97$ (dd, J = 8.5, 1.0 Hz, 1H), 6.87 (ddd, J = 8.1, 7.2, $1.2 \mathrm{~Hz}, 1 \mathrm{H}), 2.88$ (d, J = $6.5 \mathrm{~Hz}, 2 \mathrm{H}), 1.14(\mathrm{~m}, 1 \mathrm{H}), 0.63-0.58(\mathrm{~m}, 2 \mathrm{H}), 0.21(\mathrm{dd}, \mathrm{J}=10.6,5.0 \mathrm{~Hz}, 2 \mathrm{H})$.

\section{1-(2-Hydroxy-phenyl)-hept-6-en-1-one (1d phenol)}

${ }^{1} \mathrm{H}$ NMR (400 MHz, $\left.\mathrm{CDCl}_{3}\right) \delta 12.35$ (s, O프), $7.75(\mathrm{~d}, \mathrm{~J}=8.1 \mathrm{~Hz}, 1 \mathrm{H}), 7.43$ (br t, J = 8.0 Hz, 1H), $6.95(\mathrm{~d}, \mathrm{~J}=8.0 \mathrm{~Hz}, 1 \mathrm{H}), 6.86(\mathrm{t}, \mathrm{J}=8.1 \mathrm{~Hz}, 1 \mathrm{H}), 5.76(\mathrm{~m}, 1 \mathrm{H}), 4.95(\mathrm{~m}, 2 \mathrm{H})$, $2.97(\mathrm{t}, \mathrm{J}=7.4 \mathrm{~Hz}, 2 \mathrm{H}), 2.09(\mathrm{~m}, 2 \mathrm{H}), 1.75(\mathrm{~m}, 2 \mathrm{H}), 1.50(\mathrm{~m}, 1 \mathrm{H})$; GC-MS m/z $184\left(\mathrm{M}^{+}\right.$, $100 \%)$.

\section{1-(2-Hydroxy-phenyl)-3,7-dimethyl-oct-6-en-1-one (1e phenol)}

${ }^{1} \mathrm{H}$ NMR $\left(400 \mathrm{MHz}, \mathrm{CDCl}_{3}\right) \delta 12.45(\mathrm{~s}, \mathrm{OH}), 7.73(\mathrm{dd}, \mathrm{J}=7.9,1.4 \mathrm{~Hz}, 1 \mathrm{H}), 7.44(\mathrm{dt}, \mathrm{J}=$ 8.5, 1.7 Hz, 1H), $6.96(\mathrm{~d}, \mathrm{~J}=8.3 \mathrm{~Hz}, 1 \mathrm{H}), 6.86(\mathrm{t}, \mathrm{J}=7.9 \mathrm{~Hz}, 1 \mathrm{H}), 5.07$ (t, J = 7.0 Hz, 1H), $2.97(\mathrm{dd}, \mathrm{J}=15.4,5.4 \mathrm{~Hz}, 1 \mathrm{H}), 2.72(\mathrm{dd}, \mathrm{J}=15.4,8.3 \mathrm{~Hz}, 1 \mathrm{H}), 2.16(\mathrm{~m}, 1 \mathrm{H}), 2.00$ (m, 2H), 1.66 (s, 3H), $1.58(\mathrm{~s}, 3 \mathrm{H}), 1.43(\mathrm{~m}, 1 \mathrm{H}), 1.27(\mathrm{~m}, 1 \mathrm{H}), 0.96(\mathrm{~d}, \mathrm{~J}=6.7 \mathrm{~Hz}, 3 \mathrm{H})$; GC-MS $m / z 246\left(\mathrm{M}^{+}, 100 \%\right)$.

\section{Cyclopentyl-(2-hydroxy-phenyl)-methanone (1f phenol)}

${ }^{1} \mathrm{H}$ NMR $\left(400 \mathrm{MHz}, \mathrm{CDCl}_{3}\right) \delta 12.51(\mathrm{~s}, \mathrm{OH}), 7.79(\mathrm{dd}, \mathrm{J}=8.1,1.2 \mathrm{~Hz}, 1 \mathrm{H}), 7.45$ (ddd, J $=8.5,7.3,1.7 \mathrm{~Hz}, 1 \mathrm{H}), 6.97(\mathrm{dd}, \mathrm{J}=8.5,1.2 \mathrm{~Hz}, 1 \mathrm{H}), 6.88(\mathrm{dt}, \mathrm{J}=0.8,8.1 \mathrm{~Hz}, 1 \mathrm{H}), 3.74$ (m, 1H), $1.92(\mathrm{~m}, 2 \mathrm{H}), 1.75(\mathrm{~m}, 2 \mathrm{H}), 1.67$ (m, 2H); GC-MS m/z $190\left(\mathrm{M}^{+}, 100 \%\right)$.

\section{General method for the preparation of substituted 2-trifluoromethane sulfonyl} acetophenones:

Preparation of trifluoro-methanesulfonic acid 2-(cyclopent-3-enecarbonyl)-phenyl ester (1g)

Cyclopent-3-enyl-(2-hydroxy-phenyl)-methanone $(564 \mathrm{mg}, 3.0 \mathrm{mmol})$ and pyridine (475 $\mathrm{mg}, 6.0 \mathrm{mmol})$ were stirred in $\mathrm{CH}_{2} \mathrm{Cl}_{2}(15 \mathrm{~mL})$ at $-78{ }^{\circ} \mathrm{C}$ under $\mathrm{N}_{2}$. To this solution was added trifluoromethane sulfonic anhydride $(1.02 \mathrm{~g}, 3.6 \mathrm{mmol})$ in $\mathrm{CH}_{2} \mathrm{Cl}_{2}(10 \mathrm{~mL})$ dropwise over $1 / 2 \mathrm{~h}$. The mixture was allowed to warm to ambient temperature, stirred 2 $\mathrm{h}$, then poured into $1 \mathrm{~N}$ aqueous $\mathrm{HCl}$ solution $(25 \mathrm{~mL})$. The mixture was shaken, the layers were separated, and the organic layer was washed with $1 \mathrm{~N}$ aqueous $\mathrm{HCl}$ solution $(2 \times 15 \mathrm{~mL}), \mathrm{H}_{2} \mathrm{O}(2 \times 30 \mathrm{~mL})$, saturated aqueous $\mathrm{NaHCO}_{3}$ solution $(20 \mathrm{~mL})$ and finally 
saturated aqueous $\mathrm{NaCl}$ solution $(20 \mathrm{~mL})$. The organic layer was dried through a cotton plug and concentrated to an oil which was purified by chromatography on silica gel plug eluting with $10 \%$ EtOAc/hexanes to afford an oil after concentration (808 mg, 84\%). ${ }^{1} \mathrm{H}$ NMR (400 MHz, CDCl $)$ d $7.74(\mathrm{dd}, \mathrm{J}=7.8,1.7 \mathrm{~Hz}, 1 \mathrm{H}$ ), 7.56 (ddd, J = 8.3, 7.4, $1.7 \mathrm{~Hz}$, 1H), 7.45 (dd, J = 7.8, 1.2 Hz, 1H), $7.30(\mathrm{~d}, \mathrm{~J}=8.3 \mathrm{~Hz}, 1 \mathrm{H}), 5.62(\mathrm{br} \mathrm{s}, 2 \mathrm{H}), 3.95(\mathrm{~m}, 1 \mathrm{H})$, $2.77-2.58(\mathrm{~m}, 4 \mathrm{H}) ;{ }^{13} \mathrm{C} \mathrm{NMR}\left(100 \mathrm{MHz}, \mathrm{CDCl}_{3}\right) \delta 201.1,147.1,133.5,132.2,130.6$, 128.9, 128.7, 123.5, 123.1, 120.4, 117.2, 47.3, 36.0; GC-MS $m / z 320\left(\mathrm{M}^{+}, 100 \%\right)$; HRMS calcd. 321.0408 for $\mathrm{C}_{13} \mathrm{H}_{12} \mathrm{O}_{4} \mathrm{~F}_{3} \mathrm{~S}$ : obs. $\mathrm{m} / z 321.0405(\mathrm{M}+1)$.

This procedure was used to prepare the following substituted 2-trifluoromethane sulfonyl acetophenones:

\section{Trifluoro-methanesulfonic acid 2-acetyl-phenyl ester (1a)}

${ }^{1} \mathrm{H}$ NMR (400 MHz, $\left.\mathrm{CDCl}_{3}\right) \delta 7.81(\mathrm{dd}, \mathrm{J}=7.9,1.7 \mathrm{~Hz}, 1 \mathrm{H}), 7.59(\mathrm{~m}, 1 \mathrm{H}), 7.48(\mathrm{~m}, 1 \mathrm{H})$, $7.33(\mathrm{~d}, \mathrm{~J}=8.3 \mathrm{~Hz}, 1 \mathrm{H}), 2.63(\mathrm{~s}, 3 \mathrm{H}),{ }^{13} \mathrm{C} \mathrm{NMR}\left(100 \mathrm{MHz}, \mathrm{CDCl}_{3}\right) \delta$ 196.9, 147.5, 133.9, 132.3, 131.0, 128.8, 123.0, 29.7; LC-MS m/z $268\left(\mathrm{M}^{+}, 100 \%\right)$.

\section{Trifluoro-methanesulfonic acid 2-propionyl-phenyl ester (1b)}

${ }^{1} \mathrm{H}$ NMR $\left(400 \mathrm{MHz}, \mathrm{CDCl}_{3}\right) \delta 7.76(\mathrm{dd}, \mathrm{J}=7.7,1.7 \mathrm{~Hz}, 1 \mathrm{H}), 7.57(\mathrm{ddd}, \mathrm{J}=8.3,7.7,1.7$ $\mathrm{Hz}, 1 \mathrm{H}), 7.46$ (ddd, J = 7.7, 7.5, 1.0 Hz, 1H), 7.32 (d, J = 8.3 Hz, 1H), 2.96 (q, J = 7.1 Hz, $2 \mathrm{H}), 1.21(\mathrm{t}, \mathrm{J}=7.1 \mathrm{~Hz}, 3 \mathrm{H}) ;{ }^{13} \mathrm{C} \mathrm{NMR}\left(100 \mathrm{MHz}, \mathrm{CDCl}_{3}\right) \delta 201.0,146.8,133.3,132.7$, 130.2, 128.5, 122.7, 126.3, 123.1, 119.9, 116.7, 35.0, 8.0; IR ( $\left.\mathrm{cm}^{-1}\right) 3381.9,3082.2$, 2983.5, 2942.6, 2910.2, 1700.5, 1605.8, 1482.2, 1425.9, 1350.1, 1247.9, 1211.6, 1140.4, 1078.1, 956.8, 886.7, 783.7, 789.1; GC-MS $m / z 282\left(\mathrm{M}^{+}, 100 \%\right)$; HRMS calcd. 283.0252 for $\mathrm{C}_{10} \mathrm{H}_{10} \mathrm{O}_{4} \mathrm{~F}_{3} \mathrm{~S}$ : obs. $m / 2283.0252(\mathrm{M}+1)$.

\section{Trifluoro-methanesulfonic acid 2-(2-cyclopropyl-acetyl)-phenyl ester (1c)}

${ }^{1} \mathrm{H}$ NMR (400 MHz, $\left.\mathrm{CDCl}_{3}\right) \delta 7.73(\mathrm{dd}, \mathrm{J}=7.7,1.8 \mathrm{~Hz}, 1 \mathrm{H}), 7.56(\mathrm{ddd}, \mathrm{J}=8.3,7.4,1.8$ $\mathrm{Hz}, 1 \mathrm{H}), 7.45$ (ddd, J = 8.0, 7.4, 1.0 Hz, 1H), $7.32(\mathrm{~d}, \mathrm{~J}=8.3 \mathrm{~Hz}, 1 \mathrm{H}), 2.83(\mathrm{~d}, \mathrm{~J}=6.8 \mathrm{~Hz}$, $2 \mathrm{H}), 1.10(\mathrm{~m}, 1 \mathrm{H}), 0.57(\mathrm{~m}, 2 \mathrm{H}), 0.15(\mathrm{dd}, \mathrm{J}=10.6,4.8 \mathrm{~Hz}, 2 \mathrm{H})$; GC-MS $m / z 252\left(\mathrm{M}^{+}\right.$, $100 \%)$. 


\section{Trifluoro-methanesulfonic acid 2-hept-6-enoyl-phenyl ester (1d)}

${ }^{1} \mathrm{H}$ NMR (400 MHz, $\mathrm{CDCl}_{3}$ ) $\delta 7.75$ (dd, J = 7.7, $\left.1.7 \mathrm{~Hz}, 1 \mathrm{H}\right), 7.57$ (ddd, J = 8.3, 7.3, 1.9 $\mathrm{Hz}, 1 \mathrm{H}), 7.47$ (br t, J = 8.0 Hz, 1H), 7.32 (d, J = $8.3 \mathrm{~Hz}, 1 \mathrm{H}), 5.79(\mathrm{~m}, 1 \mathrm{H}), 5.00$ (dd, J = 17.1, 1.4 Hz, 1H), 4.94 (dd, J = 10.2, 1.0 Hz, 1H), 2.94 (t, J = 7.4 Hz, 2H), 2.06 (m, 2H), $1.74(\mathrm{~m}, 2 \mathrm{H}), 1.45(\mathrm{~m}, 2 \mathrm{H}) ;{ }^{13} \mathrm{C} \mathrm{NMR}\left(100 \mathrm{MHz}, \mathrm{CDCl}_{3}\right) \delta 146.4,138.4,133.3,130.3$, $130.3,128.5,128.5,122.7,114.7,41.6,33.5,28.3,23.3 ; \mathrm{CF}_{3}$ and carbonyl residues not observed; IR $\left(\mathrm{cm}^{-1}\right)$ 2934.0, 1698.5, 1605.5, 1426.7, 1216.6, 1141.1, 888.1, 769.9; GC$\mathrm{MS} m / z 267\left(\mathrm{M}-\mathrm{CF}_{3}, 100 \%\right)$.

Trifluoro-methanesulfonic acid 2-(3,7-dimethyl-oct-6-enoyl)-phenyl ester (1e)

${ }^{1} \mathrm{H}$ NMR $\left(400 \mathrm{MHz}, \mathrm{CDCl}_{3}\right) \delta 7.72(\mathrm{dd}, \mathrm{J}=7.7,1.5 \mathrm{~Hz}, 1 \mathrm{H}), 7.56$ (br t, J = 8.3 Hz, 1H), 7.45 (br dd, J = 7.7, 7.5 Hz, 1H), 7.30 (d, J = 8.1 Hz, 1H), 5.04 (m, 1H), 2.91 (dd, J = 16.7, $5.3 \mathrm{~Hz}, 1 \mathrm{H}), 2.72(\mathrm{dd}, \mathrm{J}=16.7,8.1 \mathrm{~Hz}, 1 \mathrm{H}), 2.12(\mathrm{~m}, 1 \mathrm{H}), 1.97$ (m, 2H), 1.64 (s, 3H), $1.56(\mathrm{~s}, 3 \mathrm{H}), 1.36(\mathrm{~m}, 1 \mathrm{H}), 1.24(\mathrm{~m}, 1 \mathrm{H}), 0.94(\mathrm{~d}, \mathrm{~J}=6.7 \mathrm{~Hz}, 3 \mathrm{H}) ; \mathrm{GC}-\mathrm{MS} \mathrm{m} / z, 378$ $\left(\mathrm{M}^{+}, 100 \%\right)$.

\section{Trifluoro-methanesulfonic acid 2-cyclopentanecarbonyl-phenyl ester (1f)}

${ }^{1} \mathrm{H}$ NMR $\left(400 \mathrm{MHz}, \mathrm{CDCl}_{3}\right) \delta 7.74(\mathrm{dd}, \mathrm{J}=7.7,1.7 \mathrm{~Hz}, 1 \mathrm{H}), 7.56(\mathrm{ddd}, \mathrm{J}=8.3,7.5,1.5$ $\mathrm{Hz}, 1 \mathrm{H}), 7.46$ (ddd, J = 7.7, 7.5, $1.1 \mathrm{~Hz}, 1 \mathrm{H}), 7.32$ (dd, J = 8.3, 1.1 Hz, 1H), 3.56 (quint, J $=8.0 \mathrm{~Hz}, 1 \mathrm{H}), 1.88(\mathrm{~m}, 4 \mathrm{H}), 1.71(\mathrm{~m}, 2 \mathrm{H}), 1.61(\mathrm{~m}, 2 \mathrm{H}) ;{ }^{13} \mathrm{C} \mathrm{NMR}\left(100 \mathrm{MHz}, \mathrm{CDCl}_{3}\right) \delta$ 146.8, 133.0, 132.6, 130.4, 128.4, 122.7, 122.6, 120.1, 118.6, 116.1, 49.5, 29.6, 26.1; IR $\left(\mathrm{cm}^{-1}\right)$ 2957.9, 2871.6, 1693.5, 1604.7, 1480.9, 1425.4, 1211.4, 1140.9, 999.1, 902.6, 864.2, 779.5; GC-MS m/z $322\left(\mathrm{M}^{+}, 100 \%\right)$.

\section{Trifluoro-methanesulfonic acid 2-methyl-2-propionyl-phenyl ester (1h)}

${ }^{1} \mathrm{H}$ NMR (400 MHz, $\left.\mathrm{CDCl}_{3}\right) \delta 7.69(\mathrm{dd}, \mathrm{J}=7.9,1.6 \mathrm{~Hz}, 1 \mathrm{H}), 7.56(\mathrm{~m}, 1 \mathrm{H}), 7.45(\mathrm{~m}, 1 \mathrm{H})$, $7.34(\mathrm{dd}, \mathrm{J}=8.3,0.8 \mathrm{~Hz}, 1 \mathrm{H}), 3.34(\mathrm{~m}, 1 \mathrm{H}), 1.18(\mathrm{~d}, \mathrm{~J}=6.6 \mathrm{~Hz}, 6 \mathrm{H}) ;{ }^{13} \mathrm{C} \mathrm{NMR}(100$ $\left.\mathrm{MHz}_{\mathrm{CDCl}}\right) \delta 205.0,146.9,133.2,132.2,130.3,128.7,122.9,39.2,18.6 ;$ GC-MS $\mathrm{m} / z$ $296\left(\mathrm{M}^{+}, 100 \%\right)$.

\section{Migration reaction $(1 \rightarrow 3)$}

Preparation of trifluoro-methanesulfonic acid 1-(2-hydroxy-phenyl)-propenyl ester (3b) 


\section{$\underline{t-B u O K / T H F ~ c o n d i t i o n s}$}

A solution of trifluoro-methanesulfonic acid 2-propionyl-phenyl ester (282 mg, $1.0 \mathrm{mmol})$ was stirred in anhydrous THF $(5 \mathrm{~mL})$ at $0{ }^{\circ} \mathrm{C}$ under $\mathrm{N}_{2}$ and treated with $t$-BuOK $(247 \mathrm{mg}$, $2.2 \mathrm{mmol}$ ). After $30 \mathrm{~min}$, the reaction was judged complete, poured into $1 \mathrm{~N}$ aqueous $\mathrm{HCl}$ solution $(25 \mathrm{~mL})$ at $0{ }^{\circ} \mathrm{C}$ and extracted with ethyl acetate $(3 \times 25 \mathrm{~mL})$. The organic layer was washed with $\mathrm{H}_{2} \mathrm{O}(2 \times 25 \mathrm{~mL})$, saturated aqueous $\mathrm{NaHCO}_{3}$ solution $(25 \mathrm{~mL})$ and saturated aqueous $\mathrm{NaCl}$ solution $(20 \mathrm{~mL})$. The organic layer was dried through a cotton plug and concentrated to an oil which was purified by chromatography on silica gel plug eluting with $10 \%$ EtOAc/hexanes to afford an oil after concentration $(271 \mathrm{mg}, 96 \%) .{ }^{1} \mathrm{H}$ NMR (400 MHz, $\left.\mathrm{CDCl}_{3}\right) \oint 11.09(\mathrm{~s}, \mathrm{OH}), 7.66(\mathrm{dd}, \mathrm{J}=8.2,1.4 \mathrm{~Hz}, 1 \mathrm{H}), 7.59$ (ddd, J = 7.7, 7.0, 1.4 Hz, 1H), 7.05 (dd, J = 8.5, 1.1 Hz, 1H), 6.99 (dd, J = 8.5, 7.3 Hz, 1H), 5.32 $(\mathrm{dd}, \mathrm{J}=7.0 \mathrm{~Hz}, 1 \mathrm{H}), 1.86(\mathrm{dd}, \mathrm{J}=7.0,0.8 \mathrm{~Hz}, 3 \mathrm{H})$, nOe difference experiment: irradiation of $\delta 5.32$ (vinyl proton) causes $14 \%$ enhancement of $\delta 7.66$; IR $\left(\mathrm{cm}^{-1}\right) 3055.8$, 2985.9, 1639.7, 1615.8, 1576.2, 1488.3, 1452.2, 1365.0, 1309.5, 1267.8, 1209.9, 1180.7, 1117.2, 1072.9, 1035.0, 985.0, 948.6, 828.8, 782.8, 718.3, 677.2; GC-MS m/z $282\left(\mathrm{M}^{+}\right.$, $100 \%)$.

\section{Preparation of trifluoro-methanesulfonic acid 1-(2-hydroxy-phenyl)-vinyl ester (3a) $\underline{\text { DBU/DMF conditions }}$}

Trifluoro-methanesulfonic acid 2-acetyl-phenyl ester (1a) (282 mg, $1.0 \mathrm{mmol})$ was dissolved in DMF (5.0 mL) degassed ( $3 \mathrm{~N}_{2}$ / vacuum cycles) and stirred under a $\mathrm{N}_{2}$ atmosphere. DBU (1,8-diazabicyclo[5.4.0]undec-7-ene, $380 \mathrm{mg}, 2.0 \mathrm{mmol})$ was added dropwise. The mixture was stirred at $\sim 20{ }^{\circ} \mathrm{C}$ for $18 \mathrm{~h}$, then workup up as above to provide the title compound $(274 \mathrm{mg}, 97 \%) .{ }^{1} \mathrm{H}$ NMR (400 MHz, $\left.\mathrm{CDCl}_{3}\right) \delta 11.41(\mathrm{~s}, \mathrm{OH}), 7.63(\mathrm{~m}$, 2H), $7.04(\mathrm{~m}, 2 \mathrm{H}), 4.84(\mathrm{~s}, 2 \mathrm{H}) ;{ }^{13} \mathrm{C} \mathrm{NMR}\left(100 \mathrm{MHz}, \mathrm{CDCl}_{3}\right) \oint 163.8,139.1,131.7$, 120.2, 119.7, 56.7 (not all quarternary carbons were recorded); APCI MS m/z 267 (M 1); GC-MS m/z, $268\left(\mathrm{M}^{+}, 100 \%\right)$.

Preparation of trifluoro-methanesulfonic acid 1-(2-hydroxy-phenyl)-vinyl ester (3a) $\underline{\text { KOAC/DMF conditions }}$ 
Trifluoro-methanesulfonic acid 2-acetyl-phenyl ester (1a) $(564 \mathrm{mg}, 2.0 \mathrm{mmol}$ ) was dissolved in DMF $(6.0 \mathrm{~mL})$ degassed $\left(3 \mathrm{~N}_{2} /\right.$ vacuum cycles) and stirred under a $\mathrm{N}_{2}$ atmosphere. KOAc $(589 \mathrm{mg}, 6.0 \mathrm{mmol})$ was added and the mixture was warmed to $90{ }^{\circ} \mathrm{C}$ for $30 \mathrm{~min}$, the $100{ }^{\circ} \mathrm{C}$ for $2.5 \mathrm{~h}$, cooled then workup up as above to provide the title compound (460 mg, 82\%).

\section{Preparation of trifluoro-methanesulfonic acid 1-(2-hydroxy-phenyl)-vinyl ester (3a)}

\section{LHMDS/THF conditions}

Trifluoro-methanesulfonic acid 2-acetyl-phenyl ester (1a) $(282 \mathrm{mg}, 1.0 \mathrm{mmol})$ was dissolved in THF $(2.0 \mathrm{~mL})$ and stirred under a $\mathrm{N}_{2}$ atmosphere at $-78{ }^{\circ} \mathrm{C}$. Lithium hexamethyldisilazane $(2.2 \mathrm{mmol})$ in THF $(3 \mathrm{~mL})$ was added dropwise. The mixture was stirred at $-78{ }^{\circ} \mathrm{C}$ for $1.5 \mathrm{~h}$, then poured into $1 \mathrm{~N}$ aqueous $\mathrm{HCl}$ solution $(25 \mathrm{~mL})$ and workup up as above to provide the title compound ( $262 \mathrm{mg}, 93 \%)$.

\section{Trifluoro-methanesulfonic acid 2-cyclopropyl-1-(2-hydroxy-phenyl)-vinyl ester (3c)}

Trifluoro-methanesulfonic acid 2-(2-cyclopropyl-acetyl)-phenyl ester (1c) was converted to the title compound by the $t$-BuOK method in $88 \%$ yield. ${ }^{1} \mathrm{H}$ NMR $\left(400 \mathrm{MHz}, \mathrm{CDCl}_{3}\right.$ ) $\delta 11.68$ (s, Oㅂ), $7.58(\mathrm{dd}, \mathrm{J}=8.4,7.3 \mathrm{~Hz}, 1 \mathrm{H}), 7.53$ (d, J = 8.3 Hz, 1H), 7.06 (dd, J = 8.5, $1.0 \mathrm{~Hz}, 1 \mathrm{H}), 6.97(\mathrm{ddd}, \mathrm{J}=8.1,7.3,1.0 \mathrm{~Hz}, 1 \mathrm{H}), 4.50(\mathrm{~d}, \mathrm{~J}=10.7 \mathrm{~Hz}, 1 \mathrm{H}), 1.78(\mathrm{~m}, 1 \mathrm{H})$, 1.03 (m, 1H), 0.81 (m, 2H), 0.38 (m, 1H); LC-MS m/z 120 (M-188, 100\%).

\section{Cyclization reaction $(1 \rightarrow 2)$}

\section{Preparation of 2,2-dimethyl-benzofuran-3-one (2h)}

Trifluoro-methanesulfonic acid 2-methyl-2-propionyl-phenyl ester (1h, $498 \mathrm{mg}, 1.68$ mmol) was dissolved in DMF (8.0 mL) and stirred under a $\mathrm{N}_{2}$ atmosphere. DBU $(1,8-$ diazabicyclo[5.4.0]undec-7-ene, $0.63 \mathrm{~mL}, 4.20 \mathrm{mmol}$ ) was added dropwise causing the reaction solution to become fluorescent green.. After stirring $12 \mathrm{~min}$, the reaction was judged complete, poured into $1 \mathrm{~N}$ aqueous $\mathrm{HCl}$ solution $(25 \mathrm{~mL})$ at $0{ }^{\circ} \mathrm{C}$ and extracted with ethyl acetate $(3 \times 25 \mathrm{~mL})$. The organic layer was washed with $\mathrm{H}_{2} \mathrm{O}(2 \times 25 \mathrm{~mL})$, saturated aqueous $\mathrm{NaHCO}_{3}$ solution $(25 \mathrm{~mL})$ and saturated aqueous $\mathrm{NaCl}$ solution (20 $\mathrm{mL}$ ). The organic layer was dried through a cotton plug and concentrated to an oil which was purified by chromatography on silica gel plug eluting with $10 \%$ EtOAc/hexanes to 
afford an oil after concentration $(222 \mathrm{mg}, 81 \%) .{ }^{1} \mathrm{H} \mathrm{NMR}\left(400 \mathrm{MHz}, \mathrm{CDCl}_{3}\right) \delta 7.65$ (dd, $\mathrm{J}=7.5,1.1 \mathrm{~Hz}, 1 \mathrm{H}), 7.59(\mathrm{~m}, 1 \mathrm{H}), 7.06-7.02(\mathrm{~m}, 2 \mathrm{H}), 1.44(\mathrm{~s}, 6 \mathrm{H}) ;{ }^{13} \mathrm{C} \mathrm{NMR}(100 \mathrm{MHz}$, $\left.\mathrm{CDCl}_{3}\right) \delta 171.5,138.3,125.1,121.9,119.8,113.8,88.1,23.2$; GC-MS m/z $162\left(\mathrm{M}^{+}, 83 \%\right.$, phenol, 10\%, 3-methyl-chroman-4-one, 7\%); 3-Methyl-chroman-4-one could not be separated preparatively by silica gel chromatography. It was observed by GC-MS and ${ }^{1} \mathrm{H}$ NMR (see, Crich, D.; Yao, Q. J. Org. Chem. 1995, 60, 84-88.)

\section{Preparation of 2-methyl-benzofuran-3-one (2b)}

Trifluoro-methanesulfonic acid 2-propionyl-phenyl ester (1b) $(282 \mathrm{mg}, 1.0 \mathrm{mmol})$ was dissolved in DMF (5.0 mL) degassed (3 $\mathrm{N}_{2} /$ vacuum cycles) and stirred under a $\mathrm{N}_{2}$ atmosphere. DBU (1,8-diazabicyclo[5.4.0]undec-7-ene, $380 \mathrm{mg}, 2.5 \mathrm{mmol})$ was added dropwise causing the reaction solution to become brown. The mixture was warmed to 90 ${ }^{\circ} \mathrm{C}$ with stirring for $1 \mathrm{~h}$, then worked up as above to provide product as an oil $(253 \mathrm{mg}$, 90\%). ${ }^{1} \mathrm{H}$ NMR (400 MHz, $\left.\mathrm{CDCl}_{3}\right) \delta 7.64(\mathrm{dd}, \mathrm{J}=7.7,1.3 \mathrm{~Hz}, 1 \mathrm{H}), 7.58(\mathrm{ddd}, \mathrm{J}=8.5$, 7.3, 1.5 Hz, 1H), 7.08 (d, J = 8.5 Hz, 1H), 7.05 (dd, J = 7.7, 7.3 Hz, 1H), 4.61 (q, J = 7.3 $\mathrm{Hz}, 1 \mathrm{H}), 1.50(\mathrm{~d}, \mathrm{~J}=7.3 \mathrm{~Hz}, 3 \mathrm{H}) ;{ }^{13} \mathrm{C} \mathrm{NMR}\left(100 \mathrm{MHz}, \mathrm{CDCl}_{3}\right) \delta 172.4,138.0,124.4$, 121.8, 120.4, 113.5, 81.8, 16.4; IR ( $\left.\mathrm{cm}^{-1}\right) 3200.5$, 3062.6, 2927.5, 1643.7, 1588.0, 1484.8, 1374.1, 1302.4, 1210.9, 1117.5, 990.4, 829.9, 739.6, 817.2; GC-MS m/z $148\left(\mathrm{M}^{+}, 100 \%\right)$.

\section{Preparation of 2-methyl-benzofuran-3-one (2b)}

Trifluoro-methanesulfonic acid 1-(2-hydroxy-phenyl)-propenyl ester (3b) (141 mg, 0.5 mmol) was converted to $\mathbf{2 b}$ under the above conditions except that the heating was maintained for $4 \mathrm{~h}$ for complete conversion. Workup and purification as above provided an oil (110 mg, 92\%).

\section{Preparation of 2-methyl-benzofuran-3-one (2b)}

Trifluoro-methanesulfonic acid 2-propionyl-phenyl ester (1b) (282 mg, $1.0 \mathrm{mmol})$ was converted to $\mathbf{2 b}$ using KOAc (294 $\mathrm{mg}, 3 \mathrm{mmol}$ ) as base as described above with warming to $90{ }^{\circ} \mathrm{C}$. The conversion was complete within $4 \mathrm{~h}$. Workup and purification as above provided an oil (136 mg, 92\%).

Preparation of 2-methyl-benzofuran-3-one (2b) and conversion of trifluoromethanesulfonic acid salt to trifluoromethanesulfonylmethyl-benzene 
Trifluoro-methanesulfonic acid 2-propionyl-phenyl ester (1b) $(565 \mathrm{mg}, 2.0 \mathrm{mmol})$ was dissolved in acetonitrile $(5.0 \mathrm{~mL})$ degassed $\left(3 \mathrm{~N}_{2} /\right.$ vacuum cycles) and stirred under a $\mathrm{N}_{2}$ atmosphere. DBU (1,8-diazabicyclo[5.4.0]undec-7-ene, $760 \mathrm{mg}, 5.0 \mathrm{mmol}$ ) was added dropwise. The mixture was warmed to $80{ }^{\circ} \mathrm{C}$ with stirring for $5 \mathrm{~h}$ to consume starting material, then the reaction mixture was cooled, treated with benzyl bromide $(855 \mathrm{mg}, 5$ mmol) and warmed to $80{ }^{\circ} \mathrm{C}$ with stirring for $18 \mathrm{~h}$. The reaction was cooled and worked up as above to provide product $\mathbf{2 b}(>90 \%$ by GC-MS) and after purification by chromatography on silica gel, trifluoromethanesulfonylmethyl-benzene (170 mg, 38\%) identical to authentic material. ${ }^{1} \mathrm{H}$ NMR (400 MHz, $\left.\mathrm{CDCl}_{3}\right) \delta 7.43(\mathrm{~m}, 5 \mathrm{H}), 4.47$ (s, 2H); GC-MS m/z 224 (M+1, 100\%). (See Eugene, F.; Langlois, B.; Laurent, E. J. Fluorine Chem. 1994, 66, 301-9.) 2-Methyl-benzofuran-3-one (2b) was recovered in 90\% after purification by chromatography.

\section{Preparation of 2-pent-4-enyl-benzofuran-3-one (2d)}

Trifluoro-methanesulfonic acid 2-hept-6-enoyl-phenyl ester (1d) (1.14 g, $4 \mathrm{mmol})$ was dissolved in DMF (10 mL) degassed ( $3 \mathrm{~N}_{2} /$ vacuum cycles) and stirred under a $\mathrm{N}_{2}$ atmosphere. DBU (1,8-diazabicyclo[5.4.0]undec-7-ene, $1.49 \mathrm{~mL}, 10 \mathrm{mmol})$ was added dropwise and the mixture was warmed to $90{ }^{\circ} \mathrm{C}$ with stirring for $3 \mathrm{~h}$. Workup and purification as above gave $2 \mathbf{d}(704 \mathrm{mg}, 87 \%) .{ }^{1} \mathrm{H} \mathrm{NMR}\left(400 \mathrm{MHz}, \mathrm{CDCl}_{3}\right) \delta 7.64$ (dd, J = 7.7, $0.7 \mathrm{~Hz}, 1 \mathrm{H}), 7.60(\mathrm{ddd}, \mathrm{J}=8.3,7.2,0.7 \mathrm{~Hz}, 1 \mathrm{H}), 7.10(\mathrm{dd}, \mathrm{J}=8.3,0.6 \mathrm{~Hz}, 1 \mathrm{H})$, $7.06(\mathrm{~m}, 1 \mathrm{H}), 5.77(\mathrm{~m}, 1 \mathrm{H}), 5.96(\mathrm{dd}, \mathrm{J}=17.1,1.1 \mathrm{~Hz}, 1 \mathrm{H}), 4.96(\mathrm{dd}, \mathrm{J}=10.2,1.0 \mathrm{~Hz}$, $1 \mathrm{H}), 4.55(\mathrm{dd}, \mathrm{J}=8.0,4.2 \mathrm{~Hz}, 1 \mathrm{H}), 2.10($ br q, J = 7.1 Hz, 2H), $2.02(\mathrm{~m}, 1 \mathrm{H}), 1.77(\mathrm{~m}$, 1H), $1.59(\mathrm{~m}, 2 \mathrm{H}) ;{ }^{13} \mathrm{C}$ NMR (100 MHz, $\left.\mathrm{CDCl}_{3}\right) \delta 172.8,138.0,137.9,124.3,121.8$, 121.0, 115.2, 114.5, 85.5, 33.4, 30.8, 24.0; GC-MS $m / z 202\left(\mathrm{M}^{+}, 100 \%\right)$.

\section{Preparation of 2-(1,5-dimethyl-hex-4-enyl)-benzofuran-3-one (2e)}

Trifluoro-methanesulfonic acid 2-(3,7-dimethyl-oct-6-enoyl)-phenyl ester (1e) was converted to the title compound that was obtained as an inseparable mixture of diastereomers (63/37) in $82 \%$ yield. ${ }^{1} \mathrm{H}$ NMR $\left(400 \mathrm{MHz}, \mathrm{CDCl}_{3}\right) \delta .7 .64-7.56(\mathrm{~m}, 2 \mathrm{H})$, $7.12-7.02$ (m, 2H), 5.11 (br t, J = 7.0 Hz, $\sim 0.63 \mathrm{H}$ ), 5.02 (br t, J = 7.0 Hz, $\sim 0.37 \mathrm{H}$ ), 4.52 $(\mathrm{d}, \mathrm{J}=2.9 \mathrm{~Hz}, \sim 0.63 \mathrm{H}), 4.45(\mathrm{~d}, \mathrm{~J}=2.7 \mathrm{~Hz}, \sim 0.37 \mathrm{H}), 2.23-1.88(\mathrm{~m}, 3 \mathrm{H}), 1.68(\mathrm{~s}$, $\sim 1.89 \mathrm{H}), 1.63(\mathrm{~s}, \sim 1.11 \mathrm{H}), 1.60(\mathrm{~s}, \sim 1.89 \mathrm{H}), 1.53(\mathrm{~s}, \sim 1.11 \mathrm{H}), 1.40-1.18(\mathrm{~m}, 2 \mathrm{H}), 1.09$ 
$(\mathrm{d}, \mathrm{J}=6.9 \mathrm{~Hz}, \sim 1.11 \mathrm{H}), 0.77(\mathrm{~d}, \mathrm{~J}=6.9 \mathrm{~Hz}, \sim 1.89 \mathrm{H}) ;$ GC-MS m/z $244\left(\mathrm{M}^{+}, 63.6 \%\right.$, $34.4 \%)$.

\section{Preparation of 2,2-spiropentanylbenzofuran-3-one (2f)}

Trifluoro-methanesulfonic acid 2-cyclopentanecarbonyl-phenyl ester (1f) was converted to the title compound by the method described for $2 \mathrm{~g}$ in $85 \%$ yield. ${ }^{1} \mathrm{H}$ NMR $(400 \mathrm{MHz}$, $\left.\mathrm{CDCl}_{3}\right) \delta 7.64(\mathrm{dd}, \mathrm{J}=7.0,0.8 \mathrm{~Hz}, 1 \mathrm{H}), 7.58(\mathrm{br} \mathrm{d}, \mathrm{J}=8.0,7.5 \mathrm{~Hz}, 1 \mathrm{H}), 7.04$ (dd, J = 7.3, $1.0 \mathrm{~Hz}, 1 \mathrm{H}), 7.02(\mathrm{~m}, 1 \mathrm{H}), 2.09-1.96(\mathrm{~m}, 2 \mathrm{H}), 1.95-1.89$ (m, 6H); GC-MS m/z 188 $\left(\mathrm{M}^{+}, 100 \%\right)$.

\section{Preparation of 2,2-spirocyclopentene benzofuran-3-one (2g)}

Trifluoro-methanesulfonic acid 2-(cyclopent-3-enecarbonyl)-phenyl ester (320 mg, 1.0 mmol) was dissolved in DMF (5.0 mL) degassed (3 $\mathrm{N}_{2} /$ vacuum cycles) and stirred under a $\mathrm{N}_{2}$ atmosphere. DBU (1,8-diazabicyclo[5.4.0]undec-7-ene, $380 \mathrm{mg}, 2.5 \mathrm{mmol}$ ) was added dropwise causing the reaction solution to become brown. After $10 \mathrm{~min}$, the reaction was judged complete, poured into $1 \mathrm{~N}$ aqueous $\mathrm{HCl}$ solution $(25 \mathrm{~mL})$ at $0{ }^{\circ} \mathrm{C}$ and extracted with ethyl acetate $(3 \times 25 \mathrm{~mL})$. The organic layer was washed with $\mathrm{H}_{2} \mathrm{O}(2 \times 25$ $\mathrm{mL}$ ), saturated aqueous $\mathrm{NaHCO}_{3}$ solution $(25 \mathrm{~mL})$ and saturated aqueous $\mathrm{NaCl}$ solution $(20 \mathrm{~mL})$. The organic layer was dried through a cotton plug and concentrated to an oil which was purified by chromatography on silica gel plug eluting with $10 \%$ EtOAc/hexanes to afford an oil after concentration $(70 \mathrm{mg}, 25 \%$. In another run the yield was 19\%.) ${ }^{1} \mathrm{H} \mathrm{NMR}\left(400 \mathrm{MHz}, \mathrm{CDCl}_{3}\right) \delta 7.65$ (br d, J = $\left.7.9 \mathrm{~Hz}, 1 \mathrm{H}\right), 7.58$ (ddd, J = 8.7, 7.4, 1.7 Hz, 1H), 7.05 (m, 2H), 5.77 (s, 2H), $2.77(\mathrm{AB} q, \Delta \mathrm{AB}=205.3, \mathrm{~J}=17.5 \mathrm{~Hz}, 4 \mathrm{H})$;

${ }^{13} \mathrm{C}$ NMR $\left(100 \mathrm{MHz}, \mathrm{CDCl}_{3}\right) \delta$ 204.0, 171.6, 138.3, 128.0, 124.6, 122.0, 120.7, 113.6, 95.6, 44.3; GC-MS m/z $186\left(\mathrm{M}^{+}, 100 \%\right)$; HRMS calcd. 187.0759 for $\mathrm{C}_{12} \mathrm{H}_{11} \mathrm{O}_{2}$ : obs. $\mathrm{m} / z$ $187.0766(\mathrm{M}+1)$. 


\section{Reference 13: Spiro product from Conia}

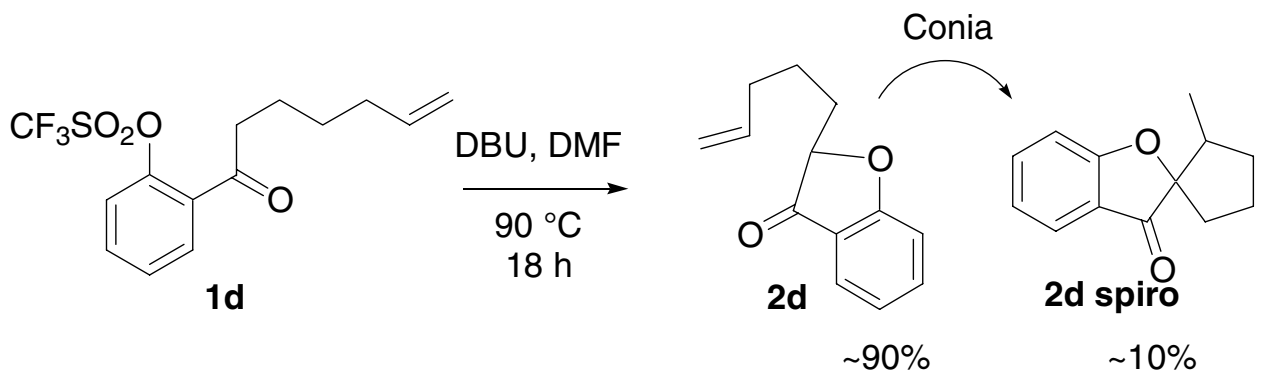

Trifluoro-methanesulfonic acid 2-hept-6-enoyl-phenyl ester (1d) $(500 \mathrm{mg}, 1.49 \mathrm{mmol})$ was dissolved in DMF $(5.0 \mathrm{~mL})$ degassed $\left(3 \mathrm{~N}_{2} /\right.$ vacuum cycles) and stirred under a $\mathrm{N}_{2}$ atmosphere. DBU (1,8-diazabicyclo[5.4.0]undec-7-ene, $0.37 \mathrm{~mL}, 3.72 \mathrm{mmol}$ ) was added dropwise. The mixture was warmed to $90{ }^{\circ} \mathrm{C}$ with stirring for $18 \mathrm{~h}$. The reaction mixture was cooled and worked up then purified as before. A minor spiro isomer of the product was isolated after purification by chromatography (24 mg, 8\%). ${ }^{1} \mathrm{H}$ NMR (400 MHz, $\left.\mathrm{CDCl}_{3}\right) \delta 7.61(\mathrm{br} \mathrm{d}, \mathrm{J}=7.7 \mathrm{~Hz}, 1 \mathrm{H}), 7.56(\mathrm{ddd}, \mathrm{J}=8.5,7.3,1.5 \mathrm{~Hz}, 1 \mathrm{H}), 7.03$ (dd, J = 8.3, $0.8 \mathrm{~Hz}, 1 \mathrm{H}), 7.02(\mathrm{~m}, 1 \mathrm{H}), 2.39(\mathrm{~m}, 1 \mathrm{H}), 2.17-1.84(\mathrm{~m}, 5 \mathrm{H}), 1.66(\mathrm{~m}, 1 \mathrm{H}), 0.91(\mathrm{~d}$, $\mathrm{J}=7.3 \mathrm{~Hz}, 3 \mathrm{H})$; GC-MS m/z $202\left(\mathrm{M}^{+}, 100 \%\right)$.

2-Pent-4-enyl-benzofuran-3-one (2d) $(50 \mathrm{mg}, 0.25 \mathrm{mmol})$ was treated under the above conditions for $18 \mathrm{~h}$. GC-MS indicated the convertion to the spiro-product was $10 \%$ complete.

\section{Preparation of Trifluoro-methanesulfonic acid 2-cyclopropyl-1-(2-methoxy-phenyl)- vinyl ester (5)}

Trifluoro-methanesulfonic acid 2-cyclopropyl-1-(2-hydroxy-phenyl)-vinyl ester (3c) (375 $\mathrm{mg}, 1.21 \mathrm{mmol}$ ) dissolved in THF ( $2 \mathrm{~mL}$ ) was added to an oil free (hexanes washed) $\mathrm{NaH}$ suspension in THF (54 mg of $60 \%$ in oil, $3.75 \mathrm{mmol}$ in $4 \mathrm{~mL} \mathrm{THF}$ ) at $0{ }^{\circ} \mathrm{C}$. After stirring $30 \mathrm{~min}$, the yellow solution was treated with methyl iodide $(0.083 \mathrm{~mL}, 1.33 \mathrm{mmol})$ and stirred for $2 \mathrm{~h}$ at $0{ }^{\circ} \mathrm{C}$ then treated with additional methyl iodide $(0.1 \mathrm{~mL})$ and let warm to ambient temperature with stirring for $18 \mathrm{~h}$. The reaction solution was treated with $\mathrm{H}_{2} \mathrm{O}$ $(10 \mathrm{~mL})$ and extracted with EtOAc $(2 \times 20 \mathrm{~mL})$. The organic layer was washed with $1 \mathrm{~N}$ aqueous $\mathrm{HCl}$ solution $(10 \mathrm{~mL}), \mathrm{H}_{2} \mathrm{O}(2 \times 10 \mathrm{~mL})$, saturated aqueous $\mathrm{NaHCO}_{3}$ solution $(25 \mathrm{~mL})$ and saturated aqueous $\mathrm{NaCl}$ solution $(20 \mathrm{~mL})$. The organic layer was dried 
through a cotton plug and concentrated to an oil which was purified by chromatography on silica gel plug eluting with 5\% EtOAc/hexanes to afford an oil after concentration (30 mg, 8\%). ${ }^{1} \mathrm{H}$ NMR (400 MHz, $\left.\mathrm{CDCl}_{3}\right) \delta 7.80(\mathrm{dd}, \mathrm{J}=7.9,1.7 \mathrm{~Hz}, 1 \mathrm{H}), 7.57$ (ddd, J = 8.2, 7.5, 1.1 Hz, 1H), 7.06 (br t, J = 8.2 Hz, 1H), 6.99 (d, J = 8.3 Hz, 1H), 5.11 (d, J = 10.3 Hz, $1 \mathrm{H}), 3.92(\mathrm{~s}, 3 \mathrm{H}), 1.68(\mathrm{~m}, 1 \mathrm{H}), 0.93(\mathrm{~m}, 1 \mathrm{H}), 0.73(\mathrm{~m}, 2 \mathrm{H}), 0.42(\mathrm{~m}, 1 \mathrm{H}) ; \mathrm{LCMS} \mathrm{m} / z$ $308.4\left(\mathrm{M}-\mathrm{CH}_{3}, 100 \%\right)$.

\section{Stability study of 4 and 5.}

2-Cyclopropyl-1-(2-methoxy-phenyl)-ethanone (4) and trifluoro-methanesulfonic acid 2-

cyclopropyl-1-(2-methoxy-phenyl)-vinyl ester (5) were independently exposed to 2.5 equiv DBU in degassed DMF at $90{ }^{\circ} \mathrm{C}$ for $48 \mathrm{~h}$ (cyclization conditions). In both cases starting material was recovered unchanged after workup in $>98 \%$ yield. Exposure to 3 equiv KOAc/DMF cyclization conditions $\left(90^{\circ} \mathrm{C}\right)$ returned 4 unchanged after $48 \mathrm{~h}$. These conditions applied to $\mathbf{5}$ provided $\mathbf{5}(70 \%)$ and $\mathbf{1 c}$ phenol $(30 \%)$. 